\title{
The finite element method for fractional non-local thermal energy transfer in non-homogeneous rigid conductors
}

\author{
Massimiliano Zingales $^{\mathrm{a}, *}$, Giuseppe Failla ${ }^{\mathrm{b}, 1}$ \\ a Department of Civil, Environmental, Aerospace and Materials Engineering (DICAM) University of Palermo, Viale delle Scienze Ed. 8, \\ 90128 Palermo, Italy \\ b Department of Civil, Energy, Environmental and Materials Engineering (DICEAM) University of Reggio Calabria, Via Graziella, \\ Località Feo di Vito, 89124 Reggio Calabria, Italy
}

\section{A R T I C L E I N F O}

\section{Article history:}

Received 3 March 2015

Revised 24 April 2015

Accepted 27 April 2015

Available online 4 May 2015

\section{Keywords:}

Fractional calculus

Finite element method

Long-range heat transport

Non-homogeneous conductors

\begin{abstract}
A B S T R A C T
In a non-local fractional-order model of thermal energy transport recently introduced by the authors, it is assumed that local and non-local contributions coexist at a given observation scale: while the first is described by the classical Fourier transport law, the second involves couples of adjacent and non-adjacent elementary volumes, and is taken as proportional to the product of the masses of the interacting volumes and their relative temperature, through a material-dependent, distance-decaying power-law function. As a result, a fractional-order heat conduction equation is derived. This paper presents a pertinent finite element method for the solution of the proposed fractional-order heat conduction equation. Homogenous and non-homogeneous rigid bodies are considered. Numerical applications are carried out on 1D and $2 \mathrm{D}$ bodies, including a standard finite difference solution for validation.
\end{abstract}

(c) 2015 Elsevier B.V. All rights reserved.

\section{Introduction}

Non-local models of thermal energy transport have been used in recent physics and engineering applications to describe "small-scale" and/or high-frequency thermodynamic processes [1-10].

Early models of non-local thermal energy transport, in rigid as well as elastic bodies, are the well-known integral models by Eringen [1-2]. They involve a thermal energy exchange with a local Fourier contribution related to the spatial gradient of the thermal energy flux, and a non-local integral term convoluting temperature gradients in the whole body with a distance-decaying attenuation function [1-2]. Models of this sort have been applied to describe thermo-elastic coupling in micro-electro-mechanical resonators [3].

In more recent years, fractional models of non-local thermal energy transport have been awarded a growing attention [4-10]. Being capable of interpolating among the integer-order operators of classical differential calculus, fractional operators have been used indeed in several contexts of physics and engineering [11-37]. They have proved suitable for reproducing long-memory effects and long-range spatial effects [20-22], with successful applications to viscoelasticity [23-28] and non-local elasticity [29-36]. Generalized transport equations have also been derived from fractional statistical mechanics [37]. Regarding, in particular, heat transfer problems in rigid as well as elastic bodies, fractional operators have been used in ref. [4-5] to model

\footnotetext{
* Corresponding author. Tel.: +39 09123896763.

1 +390965 875221.

E-mail addresses: massimiliano.zingales@unipa.it (M. Zingales), giuseppe.failla@unirc.it (G. Failla).
} 
long-memory effects, and in ref. [6-10] to model long-memory with long-range spatial effects, providing fractional time [4-6] and fractional time-space [7-9] heat conduction equations.

Very recently a non-local model of thermal energy transport has been proposed by the authors [38-41], based on the assumption that two contributions coexist at a considered observation scale: (i) a local thermal energy transfer, described by the Fourier transport law; (ii) a non-local, long-range thermal energy transport, involving couples of adjacent and non-adjacent elementary volumes, and modeled as proportional to the product of the masses of the interacting volumes, and to the relative temperature at their locations, through a material-dependent distance-decaying function. In the resulting heat conduction equation at a given location, volume integral accounts for the long-range thermal energy transfer between the given location and all surrounding adjacent and non-adjacent locations, including the material-dependent distance-decaying function that governs the long-range heat transfer [38-41]. It has been demonstrated that any strictly positive distance-decaying function can be selected, for the model to fulfill the second principle of thermodynamics [38-41]. In particular, in ref. [38-41] powerlaw distance-decaying functions have been chosen, resulting in a fractional-order heat conduction equation with some specific fractional operators [38-41]. Results have been found in agreement with non-classical thermodynamic behavior observed, for instance, in micro- and nano-devices [42-44].

This paper presents a pertinent finite element (FE) approach to solve the fractional-order heat conduction equation proposed by the authors in ref. [38-41]. Homogeneous and non-homogeneous rigid bodies are considered. Numerical results are presented for $1 \mathrm{D}$ and $2 \mathrm{D}$ rigid bodies, for different values of the fractional differentiation order. The proposed $\mathrm{FE}$ solution is validated by comparison with a standard finite difference (FD) solution.

\section{Problem formulation}

Consider an isotropic rigid body at rest and define $u=u(\mathbf{x}, t)$ as the specific internal energy, $\rho=\rho(\mathbf{x})$ as the mass density, being $\mathbf{x}=(x, y, z)$ and $t$ location and time, respectively. The mass density is not time dependent as a closed thermodynamic system is assumed. The absolute temperature of the body is denoted as $T(\mathbf{x}, t), C_{v}$ is the constant volume specific heat.

The proposed non-local model of thermodynamics [38-41] relies on the assumption that the internal energy density of the body, $\rho(\mathbf{x}) \mathbf{u}(\mathbf{x}, t)$, comprises two contributions so that:

$$
\frac{\partial \rho(\mathbf{x}) u(\mathbf{x}, t)}{\partial t}=\frac{\partial \rho(\mathbf{x}) u_{l}(\mathbf{x}, t)}{\partial t}+\frac{\partial \rho(\mathbf{x}) u_{n l}(\mathbf{x}, t)}{\partial t}
$$

In Eq. (1), $\rho(\mathbf{x}) u_{l}(\mathbf{x}, t)$ and $\rho(\mathbf{x}) u_{n l}(\mathbf{x}, t)$ denote the local and long-range contributions to the total internal energy at location $\mathbf{x}$, related to: ( $a$ ) the local flux energy vector $\mathbf{q}(\mathbf{x}, t)$; for instance, it can be provided by the Fourier law; $(b)$ a long-range residual contribution that can be expressed using fractional derivative operators [38-41].

In view of Eq. (1), the balance law of energy in presence of an energy source $\rho(\mathbf{x}) r(\mathbf{x}, t)$ is given by [38-41]:

$$
\frac{\partial \rho(\mathbf{x}) u(\mathbf{x}, t)}{\partial t}=-\nabla \mathbf{q}(\mathbf{x}, t)+\rho(\mathbf{x}) \int_{V_{\mathbf{y}}} \chi_{n l}(\mathbf{x}, \mathbf{y}, t) \rho(\mathbf{y}) d V_{\mathbf{y}}+\rho(\mathbf{x}) r(\mathbf{x}, t)
$$

where the integral on the R.H.S. is the long-range contribution due to the interactions among the elementary volume located at $\mathbf{x}$ and all other elementary volumes of the body [1-2]. In Eq. (2), $\chi_{n l}(\mathbf{x}, \mathbf{y}, t)$ is assumed as dependent on the difference between the temperatures at locations $\mathbf{x}$ and $\mathbf{y}$, in the following form:

$$
\chi_{n l}(\mathbf{x}, \mathbf{y}, t)=\frac{\kappa_{\alpha}}{d_{n, \bar{l}}(\bar{\alpha})} \frac{T(\mathbf{y}, t)-T(\mathbf{x}, t)}{\|\mathbf{x}-\mathbf{y}\|^{n+\alpha}}
$$

where $\alpha \in R, n \in N$ represents the dimension of the topological space of the body (for instance, $n=2$ for 2D bodies), and $\kappa_{\alpha}$ is a material-dependent coefficient weighing the amount of non-local heat transfer; also,

$$
\begin{aligned}
& d_{n, \bar{l}}(\bar{\alpha})=\beta_{n}(\bar{\alpha}) \frac{A_{\bar{l}}(\bar{\alpha})}{\sin (\bar{\alpha} \pi / 2)} \\
& \beta_{n}(\bar{\alpha})=\frac{\pi^{1+n / 2}}{2^{\bar{\alpha}} \Gamma(1+\bar{\alpha} / 2) \Gamma(n+\bar{\alpha} / 2)} \\
& A_{\bar{l}}(\bar{\alpha})=\sum_{k=0}^{\bar{l}}(-1)^{k-1}\left(\begin{array}{l}
\bar{l} \\
k
\end{array}\right) k^{\bar{\alpha}}
\end{aligned}
$$

where $l=\{\alpha\}+1, \bar{\alpha}=(n-1)+\alpha, \bar{l}=(n-1)+l$, being $\{\alpha\}$ the integer part of $\alpha$; obviously, Eq. (4a) holds for $\bar{\alpha} \neq 0,2,4 \ldots$, i.e. for $\bar{\alpha}$ not equal to even integers. From Eq. (2), the following fractional-order equation ruling the temperature distribution can be obtained:

$$
\rho(\mathbf{x}) C_{v} \frac{\partial T(\mathbf{x}, t)}{\partial t}=\nabla(\lambda(\mathbf{x}) \nabla T(\mathbf{x}, t))+\rho(\mathbf{x}) \frac{\kappa_{\alpha}}{d_{n, l}(\bar{\alpha})} \int_{V_{\mathbf{y}}} \frac{T(\mathbf{y}, t)-T(\mathbf{x}, t)}{\|\mathbf{x}-\mathbf{y}\|^{n+\alpha}} \rho(\mathbf{y}) d V_{\mathbf{y}}+\rho(\mathbf{x}) r(\mathbf{x}, t)
$$


where $\partial u(\mathbf{x}, t) / \partial t=C_{v} \partial T(\mathbf{x}, t) / \partial t$, Eq. (3) has been replaced for $\chi_{n l}(\mathbf{x}, \mathbf{y}, t)$ and the following Fourier linear force-flux relation has been considered for $\mathbf{q}(\mathbf{x}, t)$

$$
\mathbf{q}(\mathbf{x}, t)=-\lambda(\mathbf{x}) \cdot \nabla T(\mathbf{x}, t)
$$

being $\lambda(\mathbf{x})$ the spatially-varying local conductivity coefficient. In ref. [38-41], it has been demonstrated that no non-local terms are involved in the Neumann boundary conditions that, therefore, coincide with those of classical local thermodynamics. This is a significant advantage when numerical solutions of Eq. (5) are constructed.

It is interesting to point out that, for homogeneous bodies, the fractional-order heat conduction Eq. (5) involves well-known fractional operators. In fact, for $\lambda(\mathbf{x})=\lambda$ and $\rho(\mathbf{x})=\rho(\mathbf{y})=\rho$ over the whole domain, it can readily be seen that Eq. (5) takes the form

$$
\rho C_{v} \frac{\partial T(\mathbf{x}, t)}{\partial t}=\lambda \nabla^{2} T(\mathbf{x}, t)+\rho^{2} \kappa_{\alpha}\left(\hat{D}_{X}^{\alpha} T\right)(\mathbf{x}, t)+\rho r(\mathbf{x}, t)
$$

where $\left(\hat{D}_{X}^{\alpha} T\right)$ is the integral part of the H-Riesz fractional derivative of order $\alpha[34,35,40]$ :

$$
\left(\hat{D}_{X}^{\alpha} T\right)(\mathbf{x}, t)=\frac{1}{d_{n, \bar{l}}(\bar{\alpha})} \int_{V_{\mathbf{y}}} \frac{T(\mathbf{y}, t)-T(\mathbf{x}, t)}{\|\mathbf{x}-\mathbf{y}\|^{n+\alpha}} d V_{\mathbf{y}}
$$

In particular, for a 1D homogeneous body of uniform cross section, Eq. (7) reverts to

$$
\rho C_{v} \frac{\partial T(x, t)}{\partial t}=\lambda \frac{\partial^{2} T(x, t)}{\partial x^{2}}-\rho^{2} A \kappa_{\alpha}\left(\hat{\mathbf{D}}^{\alpha} T\right)_{x}(x, t)+\rho r(x, t)
$$

where $A$ is the area of the cross section, $\left(\hat{\mathbf{D}}^{\alpha} T\right)_{x}(x, t)=\left(\hat{\mathbf{D}}_{0+}^{\alpha} T\right)_{x}(x, t)+\left(\hat{\mathbf{D}}_{L-}^{\alpha} T\right)_{x}(x, t)$ is the sum of left and right integral parts of the Marchaud-type fractional derivatives, i.e.

$$
\begin{aligned}
& \left(\hat{\mathbf{D}}_{0+}^{\alpha} T\right)_{x}(x, t)=\frac{\alpha}{\Gamma(1-\alpha)} \int_{0}^{x} \frac{T(x, t)-T(y, t)}{(x-y)^{1+\alpha}} d y \\
& \left(\hat{\mathbf{D}}_{L-}^{\alpha} T\right)_{x}(x, t)=\frac{\alpha}{\Gamma(1-\alpha)} \int_{x}^{L} \frac{T(x, t)-T(y, t)}{(y-x)^{1+\alpha}} d y
\end{aligned}
$$

being $L$ the body length.

\section{Finite element method}

The fractional-order heat conduction Eq. (5) can be solved by standard numerical methods as the FE method. For this purpose, consider a mesh with $N_{e}$ elements over the body domain. The temperature field within the ith element is given the following form

$$
T_{i}(\mathbf{x}, t)=\mathbf{N}_{i}(\mathbf{x}) \mathbf{T}_{i}(t) \quad i=1,2, \ldots, N_{e}
$$

where $T_{i}(\mathbf{x}, t)$ is the temperature within the $i$ th element, $\mathbf{T}_{i}(t)$ is the vector of the unknown nodal temperatures of the $i$ th element, $\mathbf{N}_{i}(\mathbf{x})$ is the matrix collecting the shape functions. Upon introducing the connectivity matrix $\boldsymbol{C}_{i}$, such that

$$
\mathbf{T}_{i}(t)=\mathbf{C}_{i} \mathbf{T}(t)
$$

where $\mathbf{T}(t)$ is the vector that collects the temperatures at all nodes, replacing Eq. (11) for the temperature function in Eq. (5) and using the standard Galerkin approach along with the weak formulation [45] lead to the following equation

$$
\mathbf{M i}(t)+\left(\mathbf{K}^{(l)}+\mathbf{K}^{(n l)}\right) \mathbf{T}(t)=\mathbf{F}(t)
$$

In Eq. (13), $\mathbf{K}^{(l)}$ is the local matrix

$$
\begin{aligned}
\mathbf{K}^{(l)} & =\sum_{i=1}^{N_{e}} \mathbf{K}_{i}^{(l)} \text { for } \\
\mathbf{K}_{i}^{(l)} & =\int_{V_{i}} \sum_{s=x, y, z}\left(\mathbf{B}_{i}^{(s)}(\mathbf{x}) \mathbf{C}_{i}\right)^{\mathrm{T}} \lambda(\mathbf{x}) \mathbf{B}_{i}^{(s)}(\mathbf{x}) \mathbf{C}_{i} d V_{i}
\end{aligned}
$$

where $V_{i}$ is the volume of the $i$ th element, and $\mathbf{B}_{i}{ }^{(s)}(\mathbf{x})$, for $s=x, y, z$, is the matrix obtained by differentiating the matrix $\mathbf{N}_{i}(\mathbf{x})$ collecting the shape functions

$$
\mathbf{B}_{i}^{(s)}(\mathbf{x})=\partial \mathbf{N}_{i}(\mathbf{x}) / \partial s \quad s=x, y, z
$$

Further, in Eq. (13) $\mathbf{K}^{(n l)}$ is the non-local matrix

$$
\mathbf{K}^{(n l)}=\sum_{i=1}^{N_{e}} \mathbf{K}_{i}^{(n l)}=\sum_{i=1}^{N_{e}} \sum_{j=1}^{N_{e}} \mathbf{K}_{i j}^{(n l)} \text { for }
$$




$$
\mathbf{K}_{i j}^{(n l)}=\int_{V_{i}} \int_{V_{j}}\left(\mathbf{N}_{j}(\mathbf{y}) \mathbf{C}_{j}-\mathbf{N}_{i}(\mathbf{x}) \mathbf{C}_{i}\right)^{T} G(\mathbf{x}, \mathbf{y})\left(\mathbf{N}_{j}(\mathbf{y}) \mathbf{C}_{j}-\mathbf{N}_{i}(\mathbf{x}) \mathbf{C}_{i}\right) d V_{i} d V_{j}
$$

where $\mathbf{K}_{i j}{ }^{(n l)}$ is the non-local matrix associated with the long-range thermal energy exchange between the elementary volumes $d V_{i}$ inside the $i$ th element and those, denoted by $d V_{j}$, inside the $j$ th element, while $G(\mathbf{x}, \mathbf{y})$ is given by

$$
G(\mathbf{x}, \mathbf{y})=\frac{\kappa_{\alpha}}{d_{n, \bar{l}}(\bar{\alpha})} \frac{\rho(\mathbf{x}) \rho(\mathbf{y})}{\|\mathbf{x}-\mathbf{y}\|^{n+\alpha}}
$$

Notice that the local matrix $\mathbf{K}^{(l)}$ in Eq. (14) coincides with the classical matrix of Fourier transport, built by assembling matrices $\mathbf{K}_{i}^{(l)}$, for $i=1, \ldots N_{e}$. The non-local matrix $\mathbf{K}^{(n l)}$ in Eq. (17) is fully populated, since the terms of the non-local matrix $\mathbf{K}_{i}^{(n l)}=$ $\sum_{j=1}^{N_{e}} \mathbf{K}_{i j}^{(n l)}$, associated with the $i$ th element, will appear in all columns of the global matrix $\mathbf{K}^{(n l)}$, as can readily be seen from Eq. (18) for $\mathbf{K}_{i j}{ }^{(n l)}$.

In Eq. (13), vector $\mathbf{F}(t)$ is given as

$$
\begin{aligned}
& \mathbf{F}(t)=\sum_{i=1}^{N_{e}} \mathbf{F}_{i}(t) \text { for } \\
& \mathbf{F}_{i}(t)=\int_{V_{i}}\left(\mathbf{N}_{i}(\mathbf{x}) \mathbf{C}_{i}\right)^{\mathrm{T}} \rho(\mathbf{x}) r(\mathbf{x}, t) d V_{i}
\end{aligned}
$$

where $r(\mathbf{x}, t)$ is the vector describing internal heat sources. Eq. (20) may also contain contributions from heat flux through the boundary. In this case, vector $\mathbf{F}_{i}(t)$ associated with the $i$ th element along the boundary shall include an additional contribution given as

$$
\int_{\Gamma_{i}}\left(\mathbf{N}_{i}(\mathbf{x}) \mathbf{C}_{i}\right)^{\mathrm{T}} \bar{q} d \Gamma_{i}
$$

where $\bar{q}$ is the flux through the boundary $\Gamma_{i}$, positive outward. Also, in Eq. (13) matrix $\mathbf{M}$ is given as

$$
\begin{aligned}
& \mathbf{M}=\sum_{i=1}^{N_{e}} \mathbf{M}_{i} \text { for } \\
& \mathbf{M}_{i}=\int_{V_{i}}\left(\mathbf{N}_{i}(\mathbf{x}) \mathbf{C}_{i}\right)^{\mathrm{T}} \rho(\mathbf{x}) C_{v}\left(\mathbf{N}_{i}(\mathbf{x}) \mathbf{C}_{i}\right) d V_{i}
\end{aligned}
$$

Typical FEs for 2D heat transfer problems are either triangular or rectangular. For linear triangular elements, the matrix collecting the shape functions $\mathbf{N}_{i}(x, y)$ is a $1 \times 3$ matrix with elements given as

$$
\begin{aligned}
& N_{i 1}(x, y)=\frac{1}{2 A}\left[\left(x_{i 2} y_{i 3}-x_{i 3} y_{i 2}\right)+\left(y_{i 2}-y_{i 3}\right) x+\left(x_{i 3}-x_{i 2}\right) y\right] \\
& N_{i 2}(x, y)=\frac{1}{2 A}\left[\left(x_{i 3} y_{i 1}-x_{i 1} y_{i 3}\right)+\left(y_{i 3}-y_{i 1}\right) x+\left(x_{i 1}-x_{i 3}\right) y\right] \\
& N_{i 3}(x, y)=\frac{1}{2 A}\left[\left(x_{i 1} y_{i 2}-x_{i 2} y_{i 1}\right)+\left(y_{i 1}-y_{i 2}\right) x+\left(x_{i 2}-x_{i 1}\right) y\right]
\end{aligned}
$$

where $\left(x_{i 1}, y_{i 1}\right),\left(x_{i 2}, y_{i 2}\right)$ and $\left(x_{i 3}, y_{i 3}\right)$ are the nodal coordinates and $A$ is the area of the triangular element, i.e.

$$
A=\frac{1}{2} \operatorname{det}\left[\begin{array}{lll}
1 & x_{1} & y_{1} \\
1 & x_{2} & y_{2} \\
1 & x_{3} & y_{3}
\end{array}\right]
$$

For bilinear rectangular elements, $\mathbf{N}_{i}(x, y)$ is a $1 \times 4$ matrix with elements given as

$$
\begin{aligned}
& N_{i 1}(x, y)=\frac{1}{4 b c}(b-x)(c-y) \\
& N_{i 2}(x, y)=\frac{1}{4 b c}(b+x)(c-y) \\
& N_{i 3}(x, y)=\frac{1}{4 b c}(b+x)(c+y) \\
& N_{i 4}(x, y)=\frac{1}{4 b c}(b-x)(c+y)
\end{aligned}
$$


where $2 b$ and $2 c$ are the lengths of the $x$ - and $y$ - sides of the element (in this case, $x$ and $y$ in Eq. (27) denote local axes with origin at the center of the rectangular element).

It is worth remarking that, for 1D linear FEs, closed-form expressions can readily be derived for the integrals involved in the non-local matrices $\mathbf{K}_{i j}{ }^{(n l)}$, Eq. (18). Although in a different context, similar closed-form expressions have been provided by the authors in ref. [46].

\section{Numerical examples}

In this Section, the proposed fractional-order heat conduction Eq. (5) is solved for 1D and 2D rigid bodies, in presence of a prescribed thermal energy source $r(\mathbf{x}, t)$. Numerical solutions are built by the FE method formulated in Section 3 and compared with corresponding FD solutions.

\subsection{Temperature distribution in a $1 D$ body}

Consider the non-homogeneous rigid bar of length $L$, shown in Fig. 1 .

It is assumed that $\rho(x)=\rho$, while the local conductivity coefficient is given by

$$
\lambda(x)= \begin{cases}\lambda_{1} & -L / 2 \leq x<-\varepsilon L \\ \lambda_{1} & \varepsilon L \leq x<L / 2 \\ \lambda_{2} & -\varepsilon L \leq x<\varepsilon L\end{cases}
$$

In this case, the fractional-order heat conduction Eq. (5) takes the form (for $\rho=$ cost, see also Eq. (9)):

$$
\rho C_{v} \frac{\partial T(x, t)}{\partial t}=\frac{\partial}{\partial x}\left(\lambda(x) \frac{\partial T(x, t)}{\partial x}\right)+\rho^{2} A \kappa_{\alpha} \frac{\alpha}{\Gamma(1-\alpha)} \int_{0}^{L} \frac{T(y, t)-T(x, t)}{\|x-y\|^{1+\alpha}} d y+\rho r(x, t)
$$

The following analytical form is assumed for the thermal energy source $r(x, t)$ :

$$
r(x, t)=r_{0}\left(\langle\varepsilon L+x\rangle^{0}-\langle\varepsilon L-x\rangle^{0}\right) \cdot\left(\left\langle 1-t / \tau_{1}\right\rangle^{1}+\left\langle t / \tau_{1}-1\right\rangle^{0}+\left(e^{\left(1-t / \tau_{2}\right)}-1\right)\left\langle t / \tau_{2}-1\right\rangle^{0}\right)
$$

where $<\cdot>^{0}$ and $<\cdot>^{1}$ denote the singularity functions of order zero and one, respectively, $\tau_{1}$ and $\tau_{2}$ are reference times. That is, $r(x, t)$ in Eq. (30) spans the inner region $[-\varepsilon L, \varepsilon L]$ and exhibits a linear growth until $t=\tau_{1}$, a constant value between $\tau_{1}$ and $\tau_{2}$, and an exponential decay after $t=\tau_{2}$. A zero initial temperature is assumed over the whole bar, $T(x, 0)=0$, and zero temperatures are assumed at the boundaries for all $t$ :

$$
T(-L / 2, t)=T_{2}=0 ; \quad T(L / 2, t)=T_{1}=0
$$

To solve the fractional-order heat conduction Eq. (29), the FE approach formulated in Section 3 is applied using two-node linear elements. For comparison, a standard FD solution is built, assuming a central difference approximation for the secondorder differential operator, and a trapezoidal discretization of the integral on the R.H.S. of Eq. (29). Continuity of the heat flux is enforced at the boundaries between the inner part (with conductivity $\lambda_{2}$ ) and the outer parts (with conductivity $\lambda_{1}$ ). Considering a discrete grid of abscissas $x_{j}=(j-1) \Delta x$, with step $\Delta x=L / N$, the FD solution of Eq. (29) will be obtained at the grid points $x_{j}$ $(j=1,2, \ldots, N+1)$ from the following set of ordinary differential equations in time domain:

$$
\dot{\hat{\mathbf{T}}}(t)=\left[\hat{\mathbf{K}}^{(l)}+\hat{\mathbf{K}}^{(n l)}\right] \hat{\mathbf{T}}(t)+\overline{\mathbf{r}}(t)
$$

where $\hat{\mathbf{T}}(t)=\left[\hat{T}_{1}(t) \hat{T}_{2}(t) \ldots \hat{T}_{N+1}(t)\right]^{T}$ and $\overrightarrow{\mathbf{r}}(t)=1 / C_{v}\left[r\left(x_{1}, t\right) r\left(x_{2}, t\right) \ldots r\left(x_{N+1}, t\right)\right]^{T}$ are $(N+1)$-vectors, $\hat{\mathbf{K}}^{(l)}$ and $\hat{\mathbf{K}}^{(n l)}$ are local and non-local $(N+1) \times(N+1)$-matrices; symbol $(\wedge)$ is introduced to distinguish the FD solving equations from the FE ones in Eq. (13).

Solutions are sought for the following theoretical parameters: $L=10^{-1}, \rho=1.0, C_{v}=10^{-2}, \kappa_{\alpha}=10^{2}, \lambda_{1}=10^{-2}, \lambda_{2}=5 \times 10^{-2}$, $2 \varepsilon=10^{-1}, r_{0}=2.5 \times 10^{6} \times(2 / \pi)^{1 / 2}, \tau_{1}=10^{-4}, \tau_{2}=4 \tau_{1}$, and different values of the fractional differentiation order $\alpha$.

The FE solution obtained with $N_{e}=50$ elements is reported in Fig. 2. It can be noted that, as the long-range thermal energy transfer is introduced, the temperature distribution becomes more uniform over the bar domain and drops faster with time, with

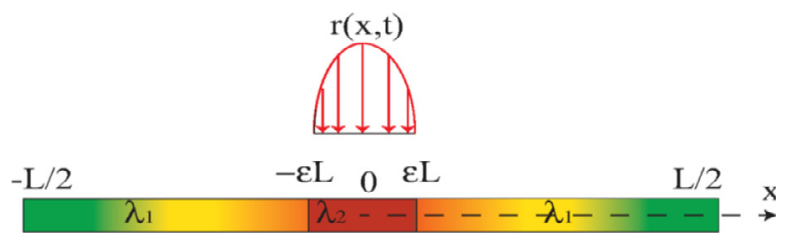

Fig. 1. 1D non-homogeneous domain. 

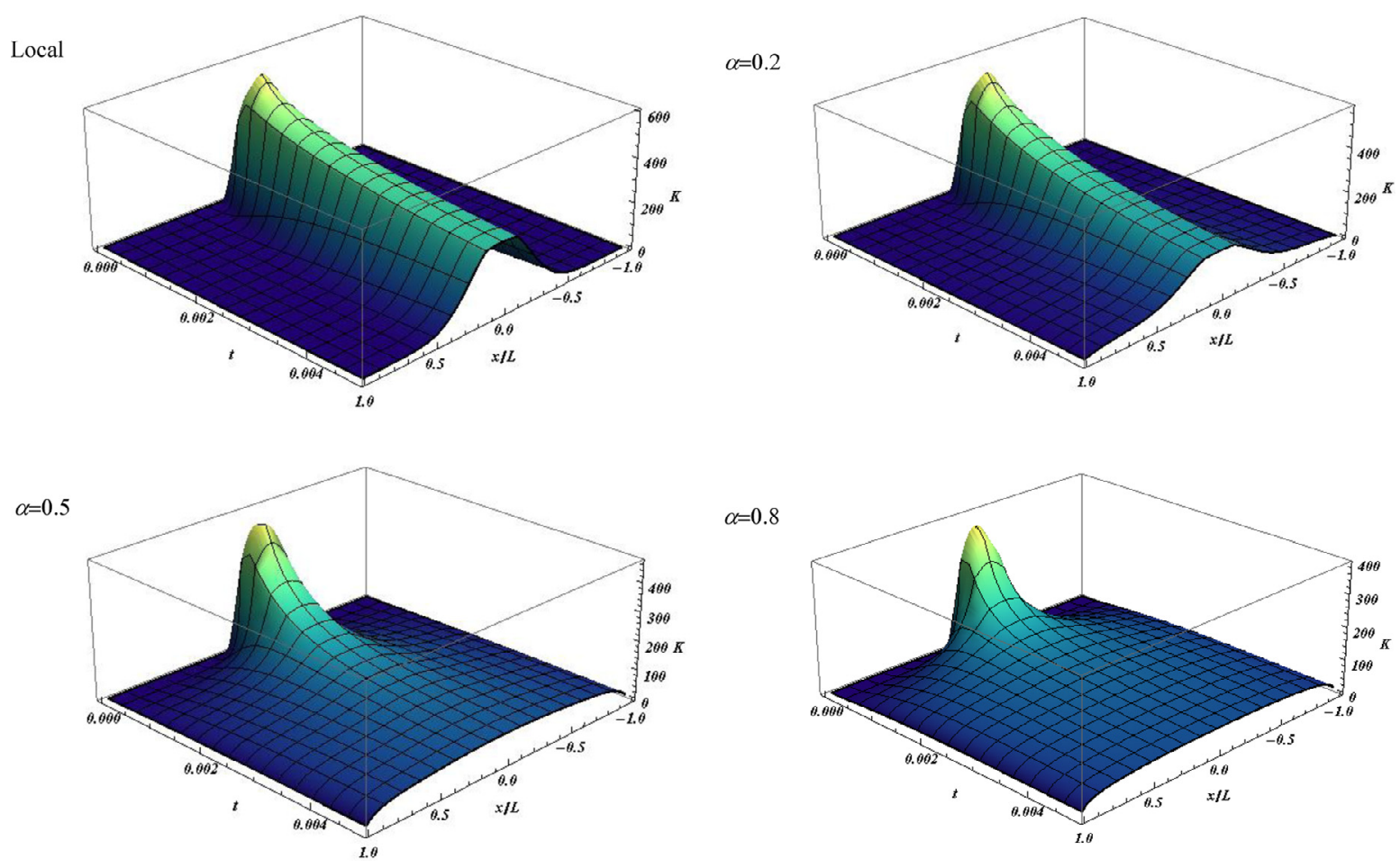

Fig. 2. Temperature field in the non-homogeneous bar (Fig. 1), for different fractional differentiation orders $\alpha$ of the long-range thermal energy transfer.

deviations from the classical local solution that vary depending on the fractional differentiation order $\alpha$. No significant changes are encountered when $N_{e}>50$ elements are used, and results are omitted for brevity.

Fig. 3 illustrates the temperature distribution across the bar at various time instants $t$, as given by the FE method with $N_{e}=50$ elements (continuous lines) and the FD method with $N=300$ intervals (symbol "•"), for different values of the fractional differentiation order $\alpha$. The two solutions are in a very good agreement, at all considered time instants.

In order to illustrate the effects of material inhomogeneity, the FE solution for the homogeneous bar, i.e. for $\lambda_{2}=\lambda_{1}$, is reported in Figs. 4 and 5. A comparison between the temperature fields in Figs. 2 and 3 (non-homogeneous bar) and in Figs. 4 and 5 (homogeneous bar) shows that, due to the higher diffusivity in the central core $\left(\lambda_{2}>\lambda_{1}\right)$, in the non-homogeneous case lower temperatures are generally experienced in the core with respect to the homogeneous case, for a given value of the fractional differentiation order $\alpha$. As a result of the long-range thermal energy transfer, however, differences become less evident as $\alpha$ increases.

\subsection{Temperature distribution in a 2D homogeneous body}

To gain a further insight into the proposed fractional-order heat conduction model, consider the 2D rigid body in Fig. 6, built by an outer square plate with a hole, welded to an inner square plate along the hole boundary. It is supposed that outer and inner plates are made of the same material, i.e. $\lambda(x, y)=\lambda$ and $\rho(x, y)=\rho$.

In this case, the heat conduction equation is Eq. (7). It is assumed that, as a result of the welding process, a thermal energy well $r(x, y, t)=r_{0} f(t)$ is uniformly distributed over a small strip of length $\Delta L$ across the hole boundary (see gray strip in Fig. 6), governed by a time-decaying function $f(t)$ :

$$
f(t)=a_{1} a_{2} \frac{e^{-a_{1} t}-e^{-a_{2} t}}{a_{2}-a_{1}}
$$

where $a_{1}$ and $a_{2}$ are real constants. A zero initial temperature is assumed over the whole domain, $T(x, y, 0)=0$, and a zero temperature is assumed along the outer sides at all $t: T(x, 0, t)=T(x, L, t)=0 ; T(0, y, t)=T(L, y, t)=0$.

To solve Eq. (7), the FE approach proposed in Section 3 is applied, with standard four-node bilinear rectangular elements. Results are compared with those from a standard FD approach, assuming a central difference approximation for the Laplace operator and a trapezoidal discretization of the integral in Eq. (7). Upon introducing a 2D node grid of $(N+1) \times(M+1)$ points (corresponding to $N$ and $M$ discretization steps along the coordinate axes $x$ and $y$, respectively), the FD solution is built from a discrete equation formally identical to Eq. (32), where in this case $\hat{\mathbf{T}}(t)$ and $\bar{r}(t)$ are $(N+1)(M+1)$-vectors, $\hat{\mathbf{K}}^{(l)}$ and $\hat{\mathbf{K}}^{(n l)}$ are $(N+1)(M+1) \times(N+1)(M+1)$-matrices. 
(a)

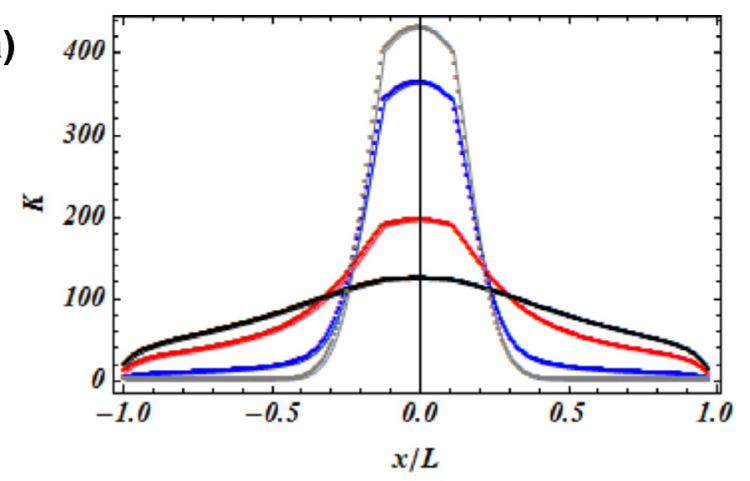

(b)

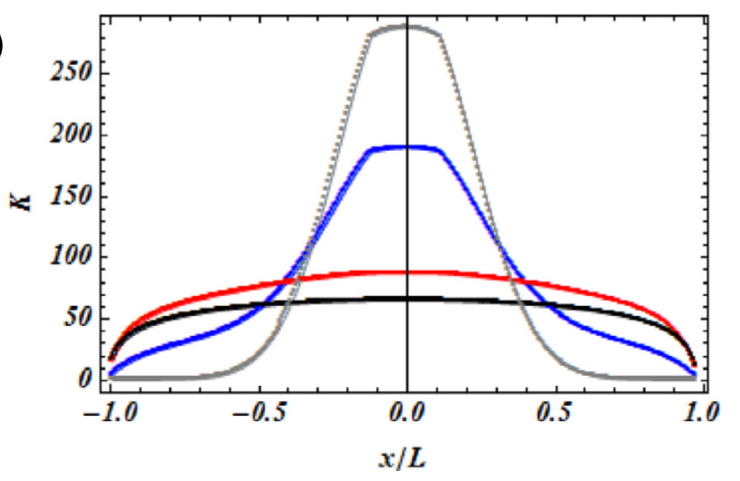

Fig. 3. Temperature distributions in the non-homogeneous bar (Fig. 1) at (a) $t=3 \times 10^{-4} \mathrm{~s}$ and (b) $t=1 \times 10^{-3} \mathrm{~s}$ : local solution (gray line), non-local solutions for $\alpha=0.2$ (blue line), $\alpha=0.5$ (red line) and $\alpha=0.8$ (black line); FE solution (continuous lines), FD solution (symbol "•"). (For interpretation of the references to colour in this figure legend, the reader is referred to the web version of this article.)
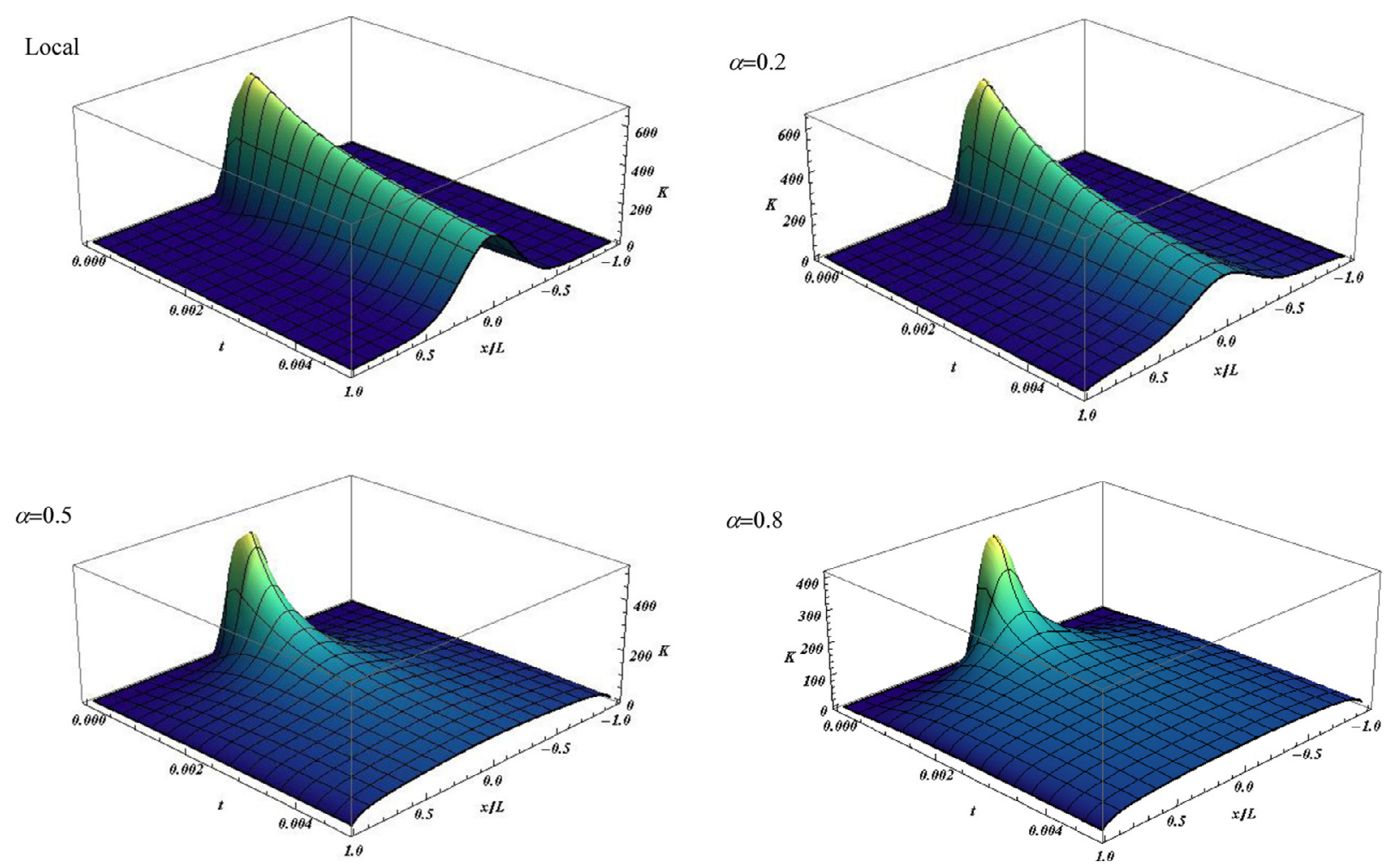

Fig. 4. Temperature field in the homogeneous bar (Fig. 1), for different fractional orders $\alpha$ of the long-range thermal energy transfer. 
(a)

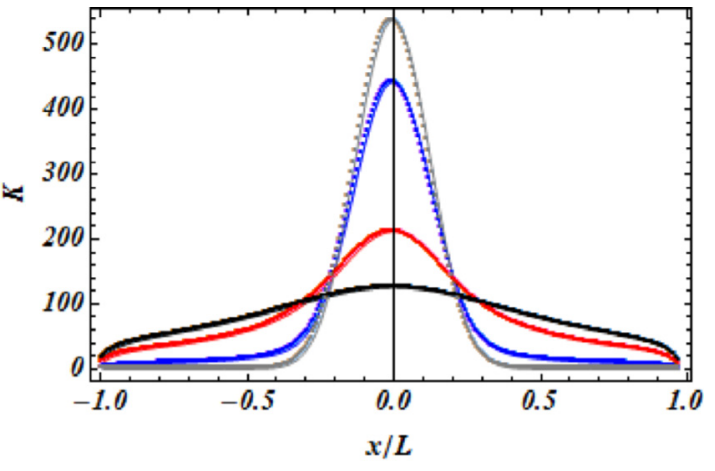

(b)

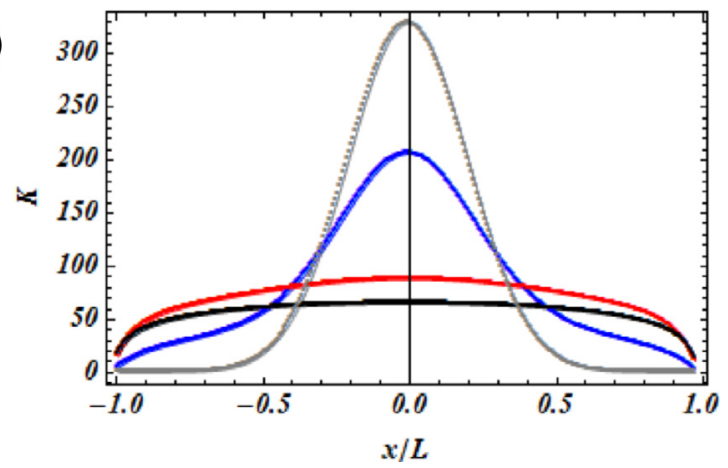

Fig. 5(a and b). Temperature distributions in the homogeneous bar (Fig. 1) at (a) $t=3 \times 10^{-4} \mathrm{~s}$ and (b) $t=1 \times 10^{-3} \mathrm{~s}$ : local solution (gray line), non-local solutions for $\alpha=0.2$ (blue line), $\alpha=0.5$ (red line) and $\alpha=0.8$ (black line); FE solution (continuous lines), FD solution (symbol "•"). (For interpretation of the references to colour in this figure legend, the reader is referred to the web version of this article.)

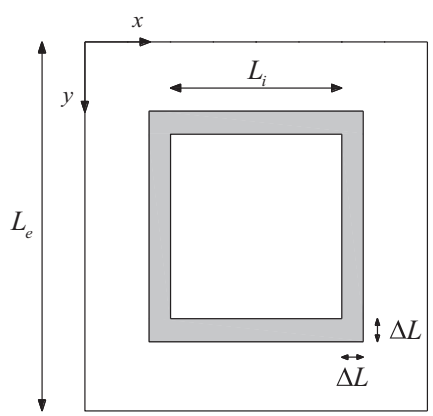

Fig. 6. 2D squared domain uniformly heated along gray contour.

The following geometry and material theoretical parameters are considered: $L_{e}=8, L_{i}=4 ; \Delta L=1$ and thickness $s=1$; $\rho=1.0, \lambda / C_{v}=0.5, \kappa_{\alpha} / d_{n, \bar{l}}(\bar{\alpha})=0.5, r_{0}=10^{4}, a_{1}=1.0$ and $a_{2}=2.0$ for $f(t)$ given by Eq. (33); $\alpha=0.1$ and $\alpha=0.7$ are considered as fractional differentiation orders.

Fig. 7 presents the FE solution when no long-range thermal energy exchange is considered, i.e. for the Fourier classical diffusion, while Figs. 8 and 9 show the FE solution of the proposed fractional-order heat conduction equation, for $\alpha=0.7$ and $\alpha=0.1$ respectively; to build the FE solutions $N_{e}=20 \times 20=400$ rectangular elements are used. It is evident that, as a result of the long-range thermal energy transfer, the temperature distribution is more uniform throughout the whole domain with respect to the case of local diffusion only, to a different degree depending on the fractional differentiation order $\alpha$. No significant changes are encountered when $N_{e}>400$ elements are used, and again results are omitted for brevity.

A further insight into the results shown in Figs. 7-9 is provided in Figs. 10 and 11, which illustrate the temperature distribution for given $y=$ cost and at various time instants $t$ (that is, lines in Figs. 10 and 11 correspond to cross sections of the temperature fields in Figs. 7-9, obtained by vertical planes at $y=$ cost). In particular, the FE solution with $N_{e}=400$ elements (continuous lines) is compared with the FD solution, obtained for $N=M=80$, i.e. totaling $81 \times 81=6561$ points in the discretization grid. The two solutions agree very well over the whole domain, at all the considered time instants. 
$t=0.25 \mathrm{~s}$
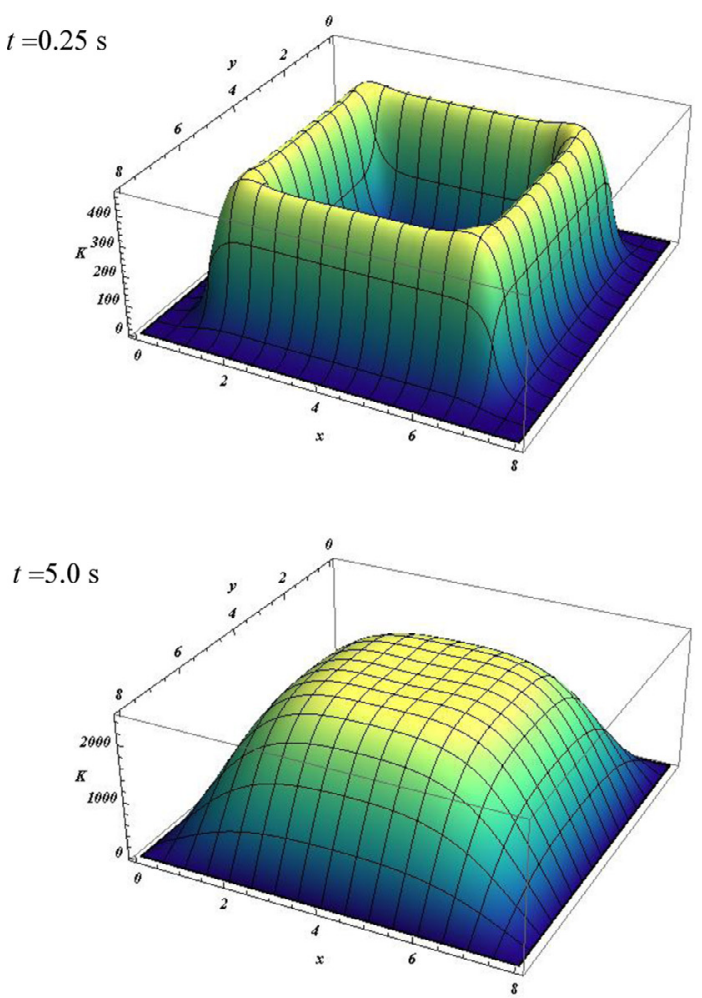

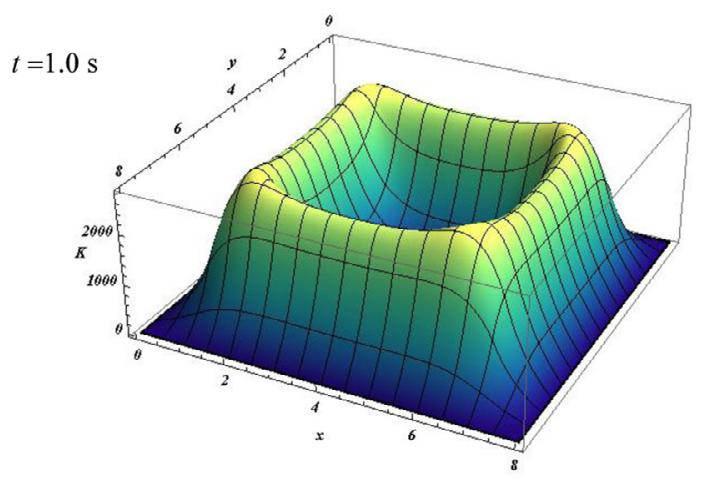

$t=7.0 \mathrm{~s}$

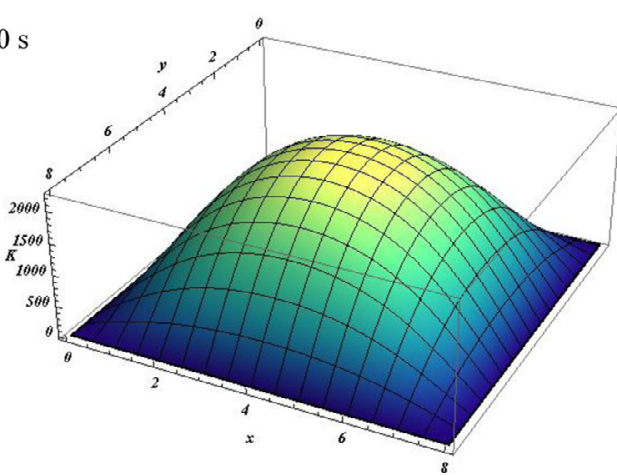

Fig. 7. Temperature field obtained with the classical Fourier transport equation $\left(\kappa_{\alpha}=0.0\right)$, at different time instants.
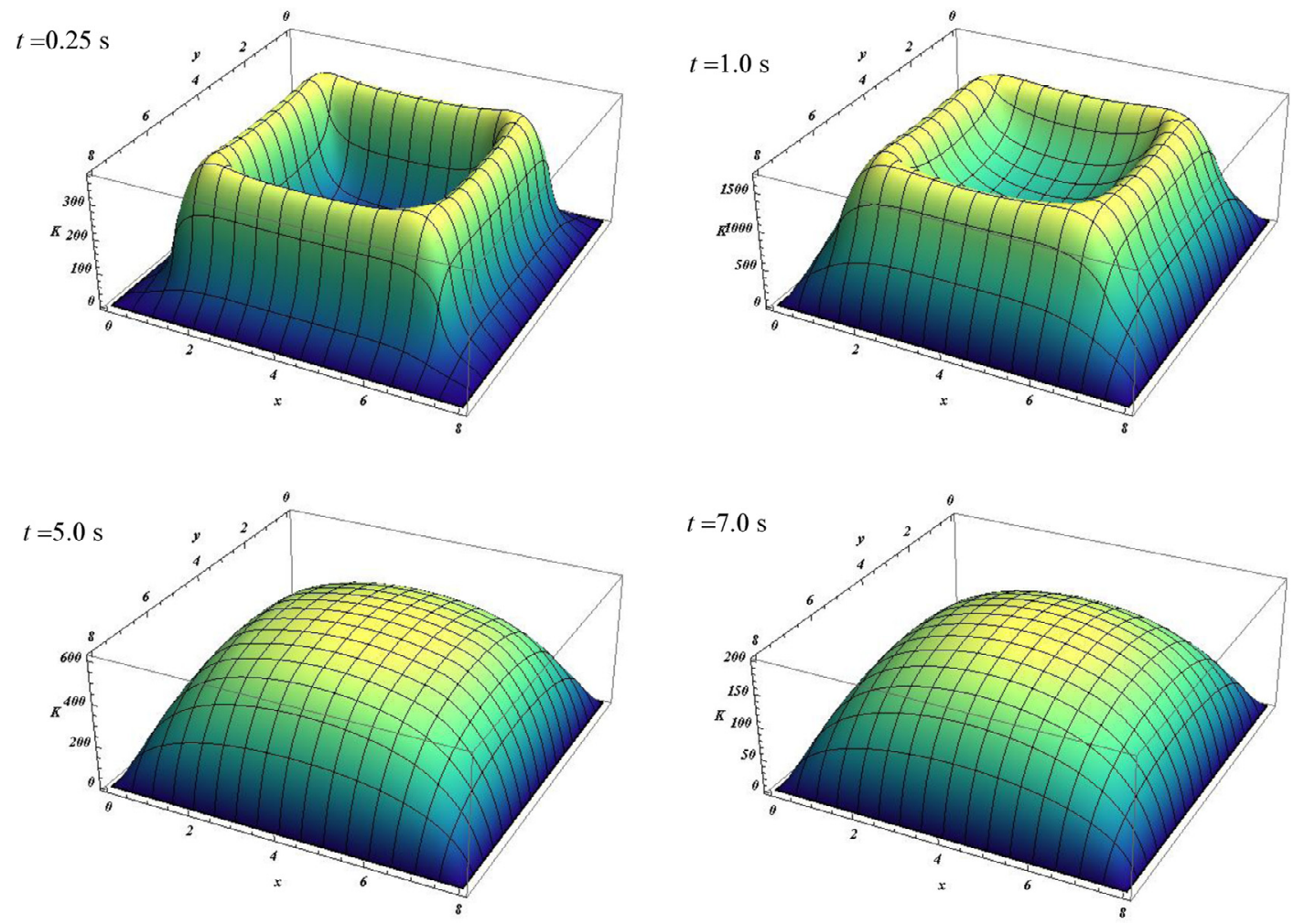

Fig. 8. Temperature field obtained with the fractional-order heat conduction equation for $\alpha=0.7$, at different time instants. 

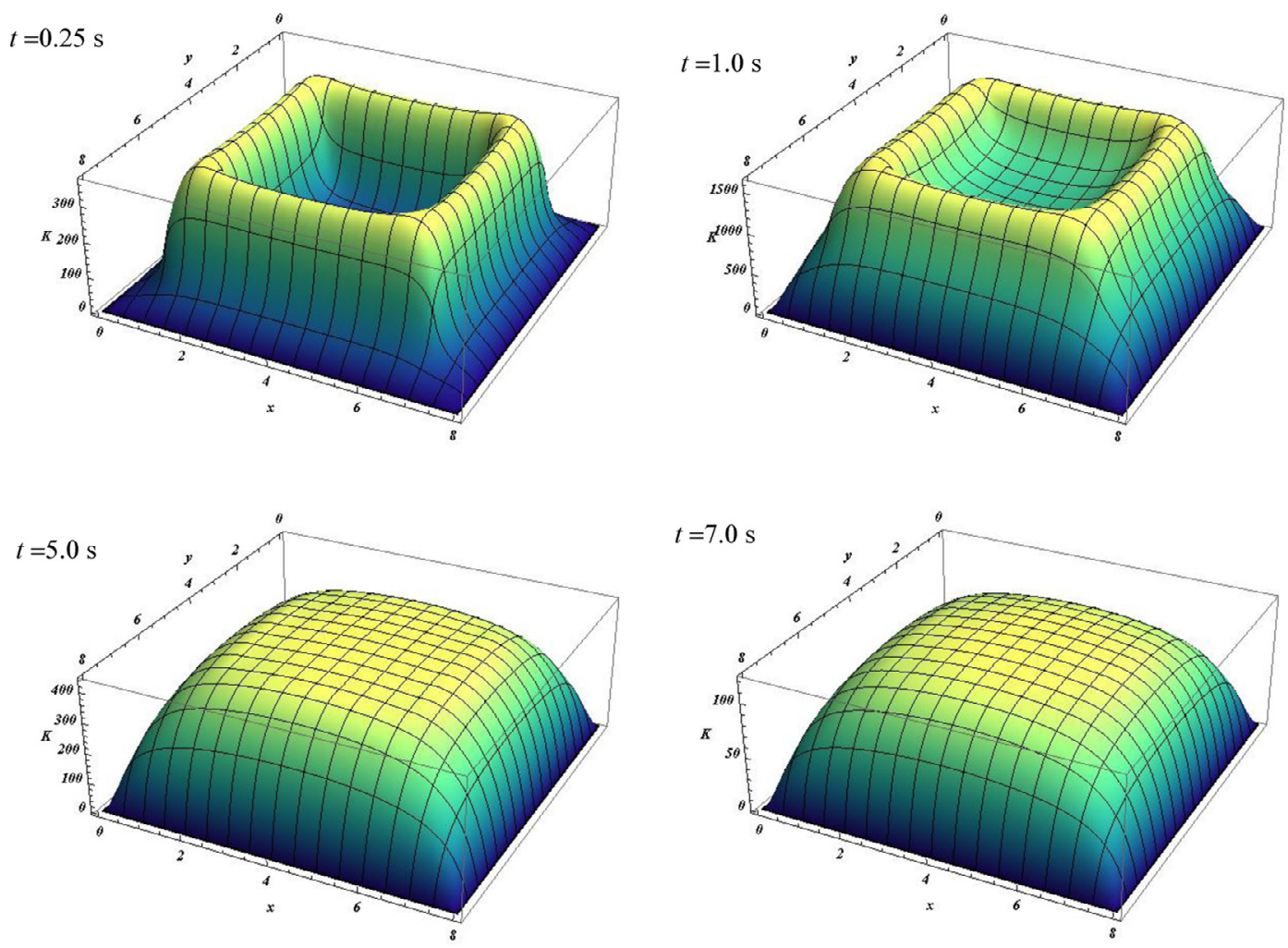

Fig. 9. Temperature field obtained with the fractional-order heat conduction equation for $\alpha=0.1$, at different time instants.

(a)

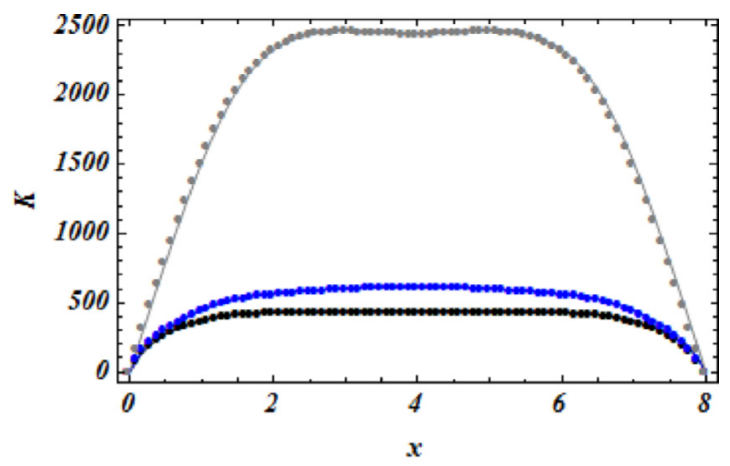

(b)

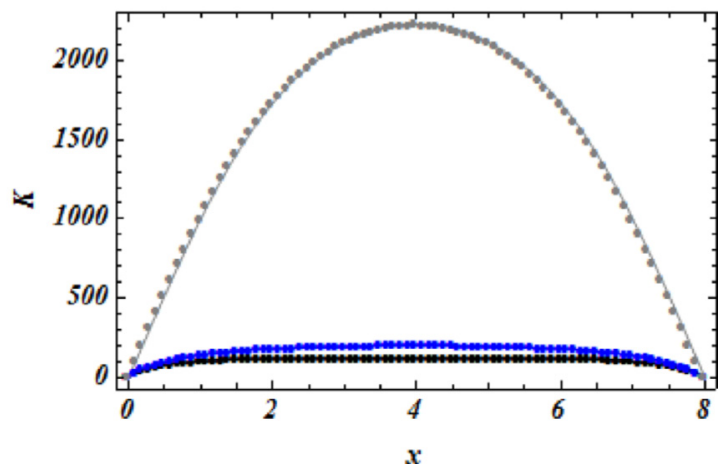

Fig. 10(a and b). Temperature distribution at $y=L_{e} / 2$ and varying $x$, for (a) $t=5.0 \mathrm{~s}$ and (b) $t=7.0 \mathrm{~s}$ : local solution (gray line), non-local solutions for $\alpha=0.7$ (blue line) and $\alpha=0.1$ (black line); FE solution (continuous lines), FD solution (symbol "•"). (For interpretation of the references to colour in this figure legend, the reader is referred to the web version of this article.) 
(a)

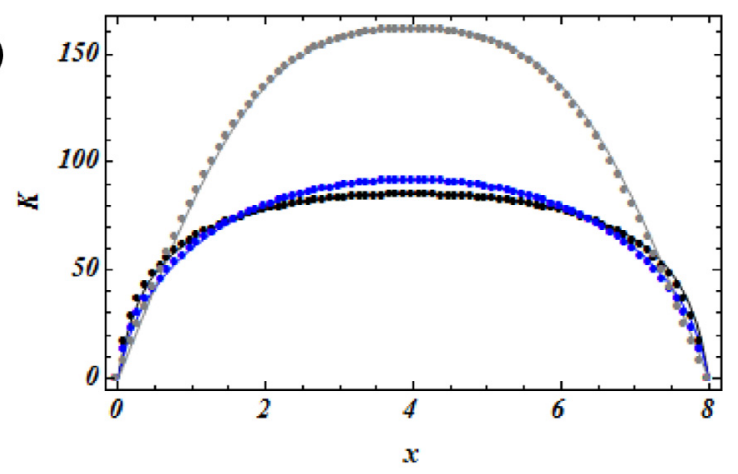

(b)

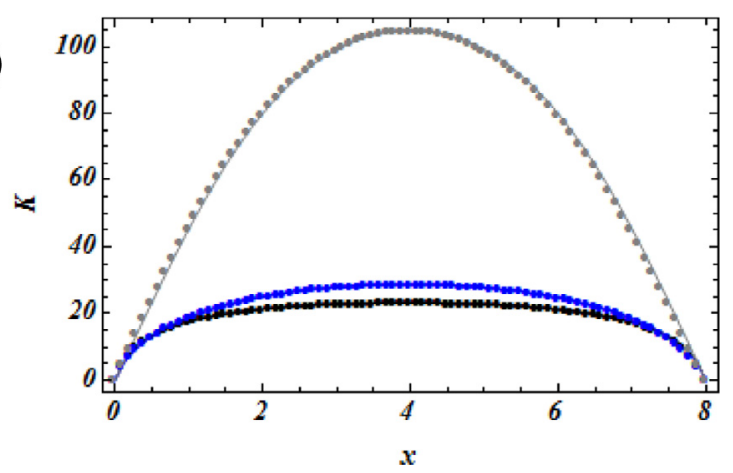

Fig. 11(a and b). Temperature distribution at $y=L_{e}-\Delta y=10^{-1}$ and varying $x$, for (a) $t=5.0 \mathrm{~s}$ and (b) $t=7.0 \mathrm{~s}$ : local solution (gray line), non-local solutions for $\alpha=0.7$ (blue line) and $\alpha=0.1$ (black line); FE solution (continuous lines), FD solution (symbol "•"). (For interpretation of the references to colour in this figure legend, the reader is referred to the web version of this article.)

\section{Conclusions}

A recent non-local, thermal energy transfer model proposed by the authors [38-41] has been investigated for homogeneous and non-homogeneous rigid bodies. The model is governed by a fractional-order heat conduction equation, which involves specific fractional operators when homogeneous bodies are considered: examples are the integral terms of the Marchaud fractional derivatives, and the integral part of the H-Riesz fractional derivatives, for 1D and 2D homogeneous bodies respectively. For either homogeneous or non-homogeneous bodies, the Neumann boundary conditions coincide with those of classical local energy transport, with significant advantages for a numerical solution of the proposed fractional-order heat conduction equation.

In particular, in this paper numerical solutions have been built by a pertinent FE method. Results have been compared with results from a standard FD method. The FE method involves a local matrix and a non-local matrix, corresponding to local and long-range thermal energy fluxes, which can readily be implemented in any numerical package. Also, closed-form solutions for the terms in the non-local matrix can be found for 1D linear FEs [46]. The comparison with the FD solution proves that accurate FE solutions can be obtained with a relatively low number of elements, and a significantly smaller size of the local and non-local matrices involved in the discretized equations (e.g., Eq. (13) and Eq. (32)) for the FE and FD methods, respectively).

The numerical applications involve 1D and 2D temperature fields induced by a thermal energy source. As expected, they show that a more uniform temperature distribution is obtained in the body domain as non-local energy transfer phenomena are accounted for. The proposed fractional-order heat conduction equation proves potentially capable of representing a large variety of non-local behaviors, depending on the parameters weighing the amount of non-local energy transfer, namely the fractional differentiation order $\alpha$ and parameter $\kappa_{\alpha}$ pre-multiplying the fractional integrals (see Eq. (5) and Eq. (7)). For its versatility, the proposed fractional model of non-local thermal energy transport may represent, in the authors' opinion, a useful tool to capture non-local deviations from the classical Fourier transport.

For completeness, the authors wish to point out that the proposed fractional-order heat conduction equation can be solved also by alternative FD approaches, which are pertinent to fractional operators, as shown in previous publications [47]. At any rate, for its simplicity and computational efficiency, the proposed FE solution appears as most suitable for a straightforward implementation by any user.

\section{References}

[1] Eringen CA. A unified theory of thermomechanical materials. Int J Eng Sci 1966;4:179-202.

[2] Eringen CA. Theory of nonlocal thermoelasticity. Int J Solids Struct 1974;12:1063-77.

[3] Ardito R, Comi C. Nonlocal thermoelastic damping in microelectromechanical resonators. J Eng Mech 2009;135:214-20. 
[4] Sherief HH, El-Sayed A, Abd-El-Latief A. Fractional order theory of thermoelasticity. Int J Solids Struct 2010;47:269-75.

[5] Youssef HM. Theory of fractional-order generalized thermoelasticity. J Heat Transf 2010;132(6):1-7.

[6] Povstenko YZ. Two-dimensional axisymmetric stresses exerted by instantaneous pulses and sources of diffusion in an infinite space in case of time-fractional diffusion equation. Int J Solids Struct 2007:44:2324-48.

[7] Povstenko YZ. Fractional heat conduction equations and associated thermal stress. J Therm Stress 2005;28:83-102.

[8] Povstenko YZ. Theory of thermoelasticity based on space-time fractional heat conduction equation. Phys Scr T136 2009:014017.

[9] Fil'Shtinskii LA, Kirichok TA, Kushnir DV. One-dimensional fractional quasi-static thermoelasticity problem for a half-space. WSEAS Trans Heat Mass Transf $2013 ; 8(2): 31-6$.

[10] Garbai L, Barna L, Bartal I, Méhes S. On the system theory of the heat flux. Two input problems. Application of fractional differential and integral operators. WSEAS Trans Heat Mass Transf 2006;1(4):506-15.

[11] Samko SG, Kilbas AA, Marichev OI. Fractional integrals and derivatives. Amsterdam: Gordon Breach; 1989.

[12] Podlubny I. Fractional differential equations. New-York: Academic Press; 1998.

[13] Padovan J. Computational algorithms for FE formulations involving fractional operators. Comput Mech 1987;2:271-87.

[14] Padovan J, Sawicki JT. Diophantine type fractional derivative representation of structural hysteresis, Part I: formulation. Comput Mech 1997;19:335-40.

[15] Rabei EM, Ajlouni AW, Ghassib HB. Quantization of nonconservative systems using fractional calculus. WSEAS Trans Math $2006 ; 5$ (7):853-63.

[16] Choudhary SK. Stability and performance analysis of fractional order control systems. WSEAS Trans Syst and Control 2014;9:438-44.

[17] Sauga A, Mankin R, Ainsaar A. Noisy fractional oscillator: temporal behavior of the autocorrelation function. WSEAS Trans Syst 2010;9(10):1019-28.

[18] Wollscheid D, Lion A. The benefit of fractional derivatives in modelling the dynamics of filler-reinforced rubber under large strains: a comparison with the Maxwell-element approach. Comput Mech 2014;53:1015-31.

[19] Birk C, Song C. An improved non-classical method for the solution of fractional differential equations. Comput Mech 2010;46:721-34.

[20] Tarasov VE, Zaslavsky GM. Conservation laws and Hamilton's equations for systems with long-range interaction and memory. Commun Nonlinear Sci Numer Simul 2008;13(9):1860-78.

[21] Tarasov VE, Zaslavsky GM. Fractional dynamics of systems with long-range space interaction and temporal memory. Phys A 2007;383:291-308.

[22] Tarasov VE. Power-law spatial dispersion from fractional Liouville equation. Phys Plasmas 2013;20(10):102110.

[23] Bagley RL, Torvik PJ. On the fractional calculus model of viscoelastic behavior. J Rheol 1986;30(1):133-55.

[24] Mainardi F. Fractional calculus and waves in linear viscoelasticity: an introduction to mathematical models. Singapore: World Scientific; 2010.

[25] Meral FC, Royston TJ, Magin R. Fractional calculus in viscoelasticity: an experimental study. Commun Nonlinear Sci Numer Simul 2010;15(4):939-45.

[26] Failla G, Pirrotta A. On the stochastic response of a fractionally-damped duffing oscillator. Commun Nonlinear Sci Numer Simul 2012;17:5131-42.

[27] Di Paola M, Failla G, Pirrotta A. Stationary and non-stationary stochastic response of linear fractional viscoelastic systems. Probab Eng Mech 2012;28:85-90.

[28] Galucio AC, Deu JF, Ohayon R. Finite element formulation of viscoelastic sandwich beams using fractional derivative operators. Comput Mech 2004;33:28291.

[29] Gubenko VS. Some contact problems of the theory of elasticity and fractional differentiation. J Appl Math Mech 1957;21(2):279-80.

[30] Tarasov VE, Aifantis EC. Non-standard extensions of gradient elasticity: fractional non-locality, memory and fractality. Commun Nonlinear Sci Numer Simul 2015;22(1-3):197-227.

[31] Tarasov VE. Lattice with long-range interaction of power-law type for fractional nonlocal elasticity. Int J Solids Struct 2014;51(15-16):2900-7.

[32] Sumelka W, Blaszczyk T. Fractional continua for linear elasticity. Arch Mech 2014;66(3):147-72.

[33] Drapaca CS, Sivaloganathan S. A fractional model of continuum mechanics. J Elast 2012;107:105-23.

[34] Failla G, Santini A, Zingales M. A non-local two-dimensional foundation model. Arch Appl Mech 2013;83:253-72.

[35] Failla G, Santini A, Zingales M. Solution strategies for 1D elastic continuum with long-range interactions: smooth and fractional decay. Mech Res Commun 2010;37:13-21.

[36] Challamel N, Zorica D, Atanacković TM, Spasić DT. On the fractional generalization of Eringen's nonlocal elasticity for wave propagation. C R Mec 2013;341:298-303.

[37] Tarasov VE. Transport equations from Liouville equations for fractional systems. Int J Mod Phys B 2006;20(3):341-53.

[38] Borino G, Di Paola M, Zingales M. A non-local model of fractional heat conduction in rigid bodies. Eur Phys J: Spec Top 2010;193:173-84.

[39] Mongioví MS, Zingales M. A non-local model of thermal energy transport: the fractional temperature equation. Int J Heat Mass Transf 2013;67:593-601.

[40] Zingales M, Failla G, Rizzo U. Fractional-order thermal energy transport for small-scale engineering devices. J Nanomech Micromec 2014;4(1):A4013006.

[41] Zingales M. Fractional-order theory of heat transport in rigid bodies. Commun Nonlinear Sci Numer Simul 2014;19(11):3938-53.

[42] Li Q Liu Q, Fan S. Thermal boundary resistances of carbon nanotubes in contact with metals and polymers. Nano Lett 2009;9(11):3805-9.

[43] Norris PM, Hopkins PE. Examining interfacial diffuse phonon scattering through transient thermoreflectance measurements of thermal boundary conductance. J Heat Transf 2009;131(4):043207.

[44] Timofeeva EV, Routbord JL, Singh D. Particle shape effects on thermophysical properties of alumina nanofluids. J Appl Phys $2009 ; 106(1): 014304$.

[45] Kwon YW, Bang H. The finite element method using Matlab. Boca Raton: CRC Press; 1997.

[46] Alotta G, Failla G, Zingales M. Finite element method for a nonlocal Timoshenko beam model. Finite Elem Anal Des 2014;89:77-92.

[47] Di Paola M, Zingales M. Fractional differential calculus for 3D mechanically based non-local elasticity. Int J Multiscale Comput Eng 2011;9(5):579-97. 